\title{
A SEMI-ANALYTIC APPROACH TO EULER SINGULARITIES *
}

\author{
RUSSEL E. CAFLISCH ${ }^{\dagger}$ AND MICHAEL SIEGEL $\ddagger$
}

In Honor of George Papanicolaou's 60th Birthday

\begin{abstract}
This paper reviews the efforts to construct (or prove the impossibility of) singular solutions of the Euler equations for three dimensional incompressible flow. A semi-analytic approach to this problem is formulated based on numerical computation of complex traveling wave solutions, followed by perturbation construction of a real solution, for axisymmetric flow with swirl. The perturbation analysis depends on small amplitude of the singularity in the traveling wave solution, for small swirl in the underlying background flow.
\end{abstract}

Key words. Euler equations, incompressible flow, singularity,multi-precision, complex traveling wave

AMS subject classifications. Primary 82D37; Secondary 82D25

1. Introduction. The possibility of singularity formation for the 3D Euler equations of incompressible inviscid fluid flow has been an open problem of mathematics and physics for over 50 years. Euler singularities were first proposed by Lars Onsager in 1949 [35] as a central mechanism in fluid dynamic turbulence. He suggested that a sufficiently strong Euler singularity could produce an inviscid dissipation mechanism in the sense that energy conservation would then be violated. He showed that inviscid energy dissipation could occur only if the singularity exponent $\alpha$ (i.e. Holder exponent) for the velocity field was no larger than $1 / 3$. This result was rigorously derived by Constantin, E and Titi [16] and Eyink [24], and the criterion was generalized by Eyink [25] and Caflisch et al. [10] to $\alpha+\kappa / 3 \leq 1 / 3$ in which $\kappa$ is the co-dimension of the singularity set.

The numerical search for singularities was initiated by Orszag and co-workers [8], who performed numerical simulation for the Taylor-Green problem using a pade expansion in time. Although initial results indicated a singularity at finite real time, subsequent studies have shown that there are no singularities in the Taylor-Green problem [6]. Further studies of possible singularity formation in vortex tubes, axisymmetric flow with swirl, Boussinesq flow and other configurations have been carried out numerically by $[4,5,7,23,26,29,30,32,34,36,37,38,42,44]$ and analytically in $[13,27,28,31]$. So far the none of these numerical or analytic constructions have provided completely convincing evidence for development of Euler singularities from standard initial data.

The first important analytic result on singularities in fluid flows was that of Beale, Kato and Majda [3] who showed that a singularity can only occur if the vorticity $\omega$ is not uniformly integrable in time; i.e. singularity formation at time $t_{*}$ implies that $\int^{t_{*}} \sup _{x}|\omega(x, t)| d t=\infty$. An earlier, but weaker, version of this result was derived in [2]. Constantin, Fefferman and Majda (CFM) [14] showed that either the velocity or the gradient of the direction of the vorticity must blow up at a singularity. Related

\footnotetext{
${ }^{*}$ Received August 31, 2004; accepted for publication October 25, 2004. Research supported in part by a Focused Research Grant from the NSF \#DMS-0354488.

${ }^{\dagger}$ Mathematics Department, University of California, Los Angeles, CA 90095-1555, U.S.A. (caflisch@math.ucla.edu).

${ }_{\ddagger}$ Department of Mathematical Sciences, New Jersey Institute of Technology, University Heights, Newark, NJ 07102-1982, U.S.A. (misieg@oak.njit.edu).
} 
results have been derived by $[11,12,15,17,18,19,20,33]$. In particular, Deng, Hou and $\mathrm{Yu}[21]$ derived a local version of the CFM result and applied it to several numerical candidate flows.

This paper describes an approach to construction of possible singular solutions for the Euler equations based on numerical simulation of complex traveling wave solutions, followed by a perturbation construction of singular real solution. The perturbation analysis depends on small amplitude of the singularity, and the perturbation parameter is the small swirl in the underlying flow.

Complex traveling wave solutions are discussed in Section 2 and the perturbation analysis is described in Section 3. Some numerical results are presented in Section 4 showing small amplitude of the singularity in the complex traveling wave. Conclusions and prospects for future work are discussed in Section 5 .

2. Complex Traveling Wave Solutions. Consider axi-symmetric flow with swirl in a periodic annulus. The velocity is $\mathbf{u}=\left(u_{r}, u_{\theta}, u_{z}\right)(r, z, t)$ which is rotationally invariant but has a nonzero angular component, in which $0<r_{1}<r<r_{2}$ and $0<z<2 \pi$ with periodicity, satisfying the equations

$$
\begin{array}{r}
r^{-1} \partial_{r}\left(r u_{r}\right)+\partial_{z} u_{z}=0 \\
\partial_{t} u_{z}+\mathbf{u} \cdot \nabla u_{z}+\partial_{z} p=0 \\
\partial_{t} u_{r}+\mathbf{u} \cdot \nabla u_{r}-r^{-1} u_{\theta}^{2}+\partial_{r} p=0 \\
\partial_{t} u_{\theta}+\mathbf{u} \cdot \nabla u_{\theta}+r^{-1} u_{\theta} u_{r}=0 .
\end{array}
$$

Our approach to the singularity problem is based on a steady background flow $\overline{\mathbf{u}}=$ $\left(0, \bar{u}_{\theta}, \bar{u}_{z}\right)(r)$, which is chosen to satisfy the Rayleigh criterion for instability [22]. The unstable mode is $\mathbf{u}_{1}=\hat{\mathbf{u}}_{1}(r) e^{i z+\sigma t}$, in which $\hat{\mathbf{u}}_{1}=\left(\hat{u}_{1 r}, \hat{u}_{1 \theta}, \hat{u}_{1 z}\right)$, and the unstable growth rate is $\sigma$. As pointed out by Siegel [41], such a steady flow and unstable mode can serve as the starting point for construction of a complex, upper-analytic traveling wave solution of the form $\mathbf{u}=\overline{\mathbf{u}}(r)+\mathbf{u}_{+}(r, z, t)$ in which

$$
\mathbf{u}_{+}=\sum_{k=1}^{\infty} \hat{\mathbf{u}}_{k}(r) \exp (i k(z-i \sigma t))
$$

by the traveling wave moves at speed $\sigma$ in the imaginary $z$-direction. Because the sum involves only positive wave numbers, there is only one-way coupling between wavenumbers; i.e., each wavenumber $k$ is only influenced by wave numbers $k^{\prime}$ that are less than $k$. This greatly simplifies the construction of $\mathbf{u}_{+}: \mathbf{u}_{1}$ is a linearly unstable mode for the background flow $\overline{\mathbf{u}}, \sigma$ is its unstable growth rate, and the equation for $\mathbf{u}_{k}$ has the form

$$
L_{k} \mathbf{u}_{k}=F_{k}\left(\overline{\mathbf{u}}, \hat{\mathbf{u}}_{1}, \ldots \hat{\mathbf{u}}_{k-1}\right)
$$

in which $L_{k}$ is an second order ODE operator in $r$.

Singularities in $\mathbf{u}_{+}$are numerically detected through the asymptotics of the Fourier components $\hat{u}_{k}$ for $u=u_{r}$ [43]. If

$$
\hat{u}_{k} \approx c k^{-\alpha-1} \exp \left(-i k z_{0}\right)
$$

for $\operatorname{Re} \alpha>-1$ and large $k$, then

$$
u_{+} \approx c_{1}\left(z-z_{0}\right)^{\alpha}
$$


for $z$ near the singularity $z_{0}$, where $c_{1}=-\pi c e^{-i \pi \alpha}(\sin (\pi \alpha) \Gamma(\alpha+1))^{-1}$ and $\Gamma(z)$ is the (complex) Gamma function. For this traveling wave, a shift in $t$ by an amount $t_{0}$ is equivalent to either multiplication of the $k$-th Fourier mode by a factor of $e^{\sigma k t_{0}}$ or a shift in imaginary component of the singularity point $z_{0}$ by an amount $\sigma t_{0}$. We choose this shift so that $\operatorname{Imag}\left(z_{0}\right)=0$ and refer to the resulting constant $c$ as the amplitude of the singularity.

3. Perturbation Approach. We look for a real Euler solution as the sum

$$
\mathbf{u}=\overline{\mathbf{u}}+\mathbf{u}_{+}+\mathbf{u}_{-}+\tilde{\mathbf{u}}
$$

in which $\mathbf{u}_{-}(z)=\mathbf{u}_{+}^{*}\left(z^{*}\right)$ (* indicates conjugation), and $\tilde{\mathbf{u}}$ is a remainder. Since $\overline{\mathbf{u}}$ and $\mathbf{u}_{+}+\mathbf{u}_{-}$are real, then $\mathbf{u}$ will be real if $\tilde{\mathbf{u}}$ is real. Moreover, $\overline{\mathbf{u}}, \overline{\mathbf{u}}+\mathbf{u}_{+}$and $\overline{\mathbf{u}}+\mathbf{u}_{-}$ are all exact solutions of the Euler equations, so that $\tilde{\mathbf{u}}$ satisfies a system of equations in which the forcing terms are quadratic terms such as

$$
\mathbf{u}_{+} \cdot \nabla \mathbf{u}_{-}+\mathbf{u}_{-} \cdot \nabla \mathbf{u}_{+}
$$

all of which are real. Construction of $\tilde{\mathbf{u}}$ will be based on perturbation methods. As discussed below, we will produce solutions $\mathbf{u}_{+}$and $\mathbf{u}_{-}$to be of small amplitude $\mathrm{O}(\varepsilon)$. Since the forcing terms (3.2) in the equation for $\tilde{\mathbf{u}}$ are of size $\varepsilon^{2}$, we expect $\tilde{\mathbf{u}}=O\left(\varepsilon^{2}\right)$. Validity of the expansion (3.1) up to and including the singularity time, requires an analysis showing that the singularity of $\tilde{\mathbf{u}}$ is of the same (or weaker) type as that of $\mathbf{u}_{+}$and $\mathbf{u}_{-}$, but with smaller amplitude.

Numerical construction of a complex traveling wave solution $\mathbf{u}_{+}$was carried out in [9] for pure swirling background flow $\overline{\mathbf{u}}=\left(0, \bar{u}_{\theta}, 0\right)(r)$. The resulting fit to the asymptotics of the Fourier components, as in (2.4) was remarkably good, with an singularity exponent $\alpha=-1 / 3$ so that the velocity itself becomes infinite at the singularity time. The cross-sectional streamlines for this flow are rolls, and the singularity forms approximately at the centers of the rolls. The amplitude of $\mathbf{u}_{+}$was not small, however, so that there was no possibility to create a real singular solution by a perturbation construction.

The perturbation approach to singularity formation is motivated by similar studies for vortex sheets. Siegel $[39,40]$ studied complex traveling waves for a vortex sheet separating two fluids of different densities in the Boussinesq approximation. The vorticity is measured by a vortex sheet strength $\gamma$ and the density difference (in units with the gravitational constant set to 1) by the Atwood number $A$. For the pure Boussinesq case $(A=1, \gamma=0)$ there are traveling waves of amplitude $\mathrm{O}(1)$, which is too large to allow perturbation theory. For the pure vortex sheet case $(A=0, \gamma=1)$ there are no traveling waves due to the conservation of vorticity values for $2 \mathrm{D}$ flow. On the other hand, for a vortex sheet with small Atwood number $(A<<1, \gamma=1)$, Siegel found that there are complex traveling waves with small amplitude $\varepsilon$ at the real singularity time, such that $\varepsilon \rightarrow 0$ as $A \rightarrow 0$. Using these vortex sheet results as a guide for the 3D Euler problem, we include axial flow in the background, and let the amount of swirl go to 0 . Note that in the vortex sheet problem, singularity formation is associated with ill-posedness; whereas the traveling waves solutions for Euler problem come from a balance between instability and nonlinearity.

The domain for the traveling wave solution is taken to be an annulus $r_{1}<r<r_{2}$, with $r_{1}=1$ and $r_{2}=3$, and a periodic domain in $z$ with period $2 \pi$. The background flow $\overline{\mathbf{u}}$ is taken to be a smoothed out vortex sheet on $r=r_{0}=2$, with width $d$. Let $\bar{u}_{\theta 0}$ be the background angular velocity from [9] in which there was no axial background 
flow. Let $\bar{u}_{z 0}$ be the axial velocity for a purely axial background flow, given by

$$
\begin{aligned}
\bar{u}_{z 0}(r) & =w_{1}+\left(w_{2}-w_{1}\right)\left(\tanh (s)-\tanh _{1}\right) /\left(\tanh _{2}-\tanh _{1}\right) \\
s & =\left(r-r_{0}\right) /\left(d *\left(r_{2}-r_{1}\right)\right) \\
\tanh _{i} & =\tanh \left(s_{i}\right)=\tanh \left(s\left(r=r_{i}\right)\right)
\end{aligned}
$$

The background flow is taken to be

$$
\begin{aligned}
& \bar{u}_{z}=\gamma \bar{u}_{z 0} \\
& \bar{u}_{\theta}=\Gamma \bar{u}_{\theta 0}
\end{aligned}
$$

with $\bar{u}_{r}$ determined from incompressibility. The limiting velocities $w_{1}$ and $w_{2}$ are chosen so that $w_{2}-w_{1}=1$ and that the first unstable mode for the background flow $\overline{\mathbf{u}}$ has a real growth rate $\sigma$.

$\Gamma$ and $\gamma$ are coefficients to control the strength of the angular and axial components. The extreme case $\Gamma=0$ is pure axial flow, for which the Euler equations are known to be nonsingular. In order to parameterize these coefficients, we take

$$
\begin{aligned}
& \Gamma=\cos \left(\theta_{\gamma} \pi / 2\right) \\
& \gamma=\sin \left(\theta_{\gamma} \pi / 2\right)
\end{aligned}
$$

in which the single parameter $\theta_{\gamma}$ varies between 0 and 1 .

The $k=1$ mode $\hat{\mathbf{u}}_{1}$ is the first unstable mode for the background flow $\overline{\mathbf{u}}$, and the traveling wave speed $\sigma$ has a real growth rate for this mode. The remaining Fourier coefficients $\hat{\mathbf{u}}_{k}$ are determined from the equations (2.3).

4. Numerical Results. We have performed a series of computations over a range of values of $\Gamma$ between 0 and 1 . The objective of these computations is to examine the convergence of the computational method and the dependence on $\Gamma$ for the solutions.

Because of the one-way coupling between different wavenumber in (2.3), these computations are performed for a fixed set of $z$-wavenumbers $k$ with $1 \leq k \leq n_{z}$. The number $n_{r}$ of points in $r$ is increased until the results converge. In the computations presented below, a very large value of $n_{r}$ is required, so that the maximum number $n_{z}$ of wavenumber is limited.

The numerical construction of this solution relied on solution of the system of ODEs (2.3). One difficulty was that this system was found to magnify roundoff error. This was controlled using ultra-high precision (e.g. precision $10^{-100}$ ) with the MPFUN package from David Bailey [1], which increased the computational complexity by a factor of around 100 .

Figure 1 show the results for $d=0.3, \theta_{\gamma}=0.5$ (i.e. $\left.\Gamma=1 / \sqrt{(2)}\right)$ with $n_{r}$ varying. The figures show the values of the real $\alpha$ and the imaginary part of $z_{*}$ as a function of $k$. The imaginary part of $\alpha$ was found to be approximately 0 ; so that $\alpha$ is real and approximately equal to $1 / 2$. The imaginary part of $z_{*}$ is the imaginary position of the singularity in the complex $z$-plane. A good fit is one for which the values are nearly independent of $k$ for $k$ sufficiently large. These results show that a good fit is obtained for sufficiently large values of $n_{r}$.

The amplitude $|c|$ of the singularity in the traveling wave and the norm of the traveling wave $\mathbf{u}_{+}$are plotted in Figure 2 as a function of the variable $\theta_{\gamma}$. This shows that as $\theta_{\gamma} \rightarrow 1$ (i.e., as the background flow becomes pure axial flow), the amplitude of the singularity goes to 0 , while the norm of the traveling wave stays bounded. In addition the singularity exponent $\alpha$ is nearly independent of $\theta_{\gamma}$. 

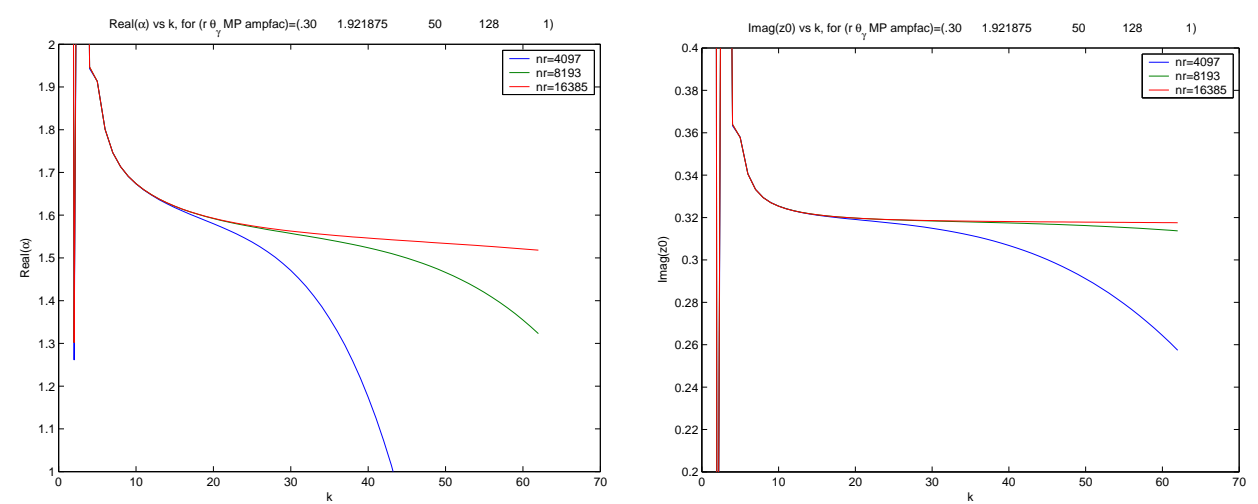

FIG. 1. Plot of the real part of $\alpha$ (left) and imaginary part of $z_{*}$ (right) for $d=0.3$ and $\theta_{\gamma}=0.5$ for different values of $n_{r}$.
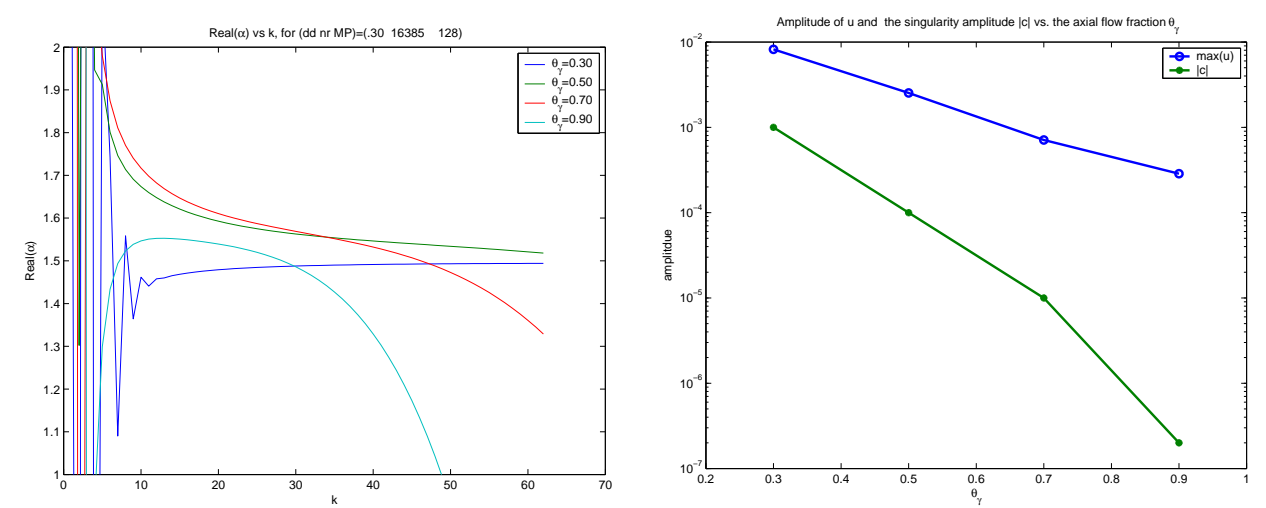

FIG. 2. Plot of the real part of $\alpha$ (left) and of the norm of $\mathbf{u}_{+}$(upper curve on the right) and the singularity amplitude $|c|$ (lower curve on right) vs. the axial flow fraction $\theta_{\gamma}$ for $d=0.3$ and $n_{r}=16,385$ for different values of $\theta_{\gamma}$.

5. Conclusions. The computations described above are limited to small values of the Fourier wavenumber $k$, due to the use of ultra-high precision, as discussed in Section 3, so that they provide limited information. Nevertheless, they illustrate an important property of the complex traveling wave solutions. As the swirl in the background flow $\overline{\mathbf{u}}$ goes to 0 , the amplitude of the singularity in the traveling wave $\mathbf{u}_{+}$ goes to zero, while the norm of $\mathbf{u}_{+}$stays bounded. Moreover, the type of singularity, as indicated by the power $\alpha$ stays roughly constant.

A puzzling aspect of these solutions is that the exponent $\alpha$ is approximately $1 / 2$ which corresponds to a singularity that is too weak to satisfy the Beale-Kato-Majda criterion. Possible resolutions of this discrepancy are that the perturbation $\tilde{u}$ has a stronger type singularity than $\mathbf{u}_{+}$or that the correct exponent $\alpha$ is not seen in the small values of $k$ that have been computed so far. In future work, we will carry these computations our for larger values of $k$ by modifying the method to remove the amplification of roundoff error. 


\section{REFERENCES}

[1] David H. BAILEY, MPFUN: A portable high performance multiprecision package, RNR Technical Report RNR-90-022, 1991.

[2] Claude Bardos and S. Benachour, Domaine d'analycite des solutions de l'equation d'Euler dans un ouvert de $R^{n}$, Annali della Scuola Normale Superiore di Pisa, IV, 4 (1977), pp. $647-687$.

[3] J. T. Beale, T. Kato And A. Majda, Remarks on the breakdown of smooth solutions for the 3-D Euler equations, Comm. Math. Phys., 94 (1984), pp. 61-66.

[4] John Bell and Daniel Marcus, Vorticity intensification and treansition to turbulence in the three dimensional Euler equations, Comm. Math. Phys., 147 (1992), pp. 327-344.

[5] A. Bhattacharjee and Xiaogang Wang, Finite-time vortex singularity in a model of threedimensional Euler flows, Phys. Rev. Lett., 69 (1992), pp. 2196-2199.

[6] M. E. BRAChet, The geometry of small-scale structures of the Taylor-Green vortex, Comptes Rendus, 311 (1990), pp. 775-780.

[7] M. E. Brachet, M. Meneguzzi, A. Vincent, H. Politaon and P. L. Sulem, Numerical evidence of a smooth self similar dynamics and possibility of subsequent collapse for three dimensional ideal flows, Phys. Fluids A, 4 (1992), pp. 2845-2854.

[8] M. E. Brachet, D. Meiron, S. Orszag, B. Nickel, R. Morf and U. Frisch, Small-scale structure of the Taylor-Green vortex, J. Fluid Mech., 130 (1983), pp. 411-452.

[9] R. E. CAflisch, Singularity formation for complex solutions of the $3 D$ incompressible Euler equations, Physica D, 67 (1993), pp. 1-18.

[10] R. E. Caflisch, I. Klapper and G. Steele, Remarks on singularities, dimension and energy dissipation for ideal hydrodynamics and MHD, Comm. Math. Phys., 184 (1997), pp. 443456.

[11] Dongho Chat, Remarks on the blow-up of the Euler equations and the related equations, Comm. Math. Phys., 245 (2003), pp. 539-550.

[12] Dongho Chat, Local existence and blow-up criterion for the Euler equations in the Besov spaces, Asymptotic Analysis, 38 (2004), pp. 339-358.

[13] S. Childress, G. R. Ierley, E. A. Spiegel and W. R. Young, Blow-up of unsteady twodimensional Euler and Navier-Stokes solutions having stagnation point form, J. Fluid Mech., 203 (1989), pp. 1-22.

[14] P. Constantin, C. Fefferman and A. J. Majda, Geometric constraints on potentially singular solutions for the 3-D Euler equations, Comm. PDE, 21 (1996), pp. 559-571.

[15] Peter Constantin, Geometric statistics in turbulence, SIAM Review, 36 (1994), pp. 73-98.

[16] Peter Constantin, Weinan E and Edriss S. Titi, Onsager's conjecture on energy conservation for solutions of Euler's equation, Comm. Math. Phys., 165 (1994), pp. 207-209.

[17] Peter Constantin and Charles Fefferman, Direction of vorticity and the problem of global regularity for the Navier-Stokes equations, Indiana U. Math. J., 42 (1994), pp. 775-789.

[18] D. Cordoba, On the geometry of solutions of the quasi-geostrophic and Euler equations, Proc. NAS, 94 (1997), pp. 12769-12770.

[19] D. Cordoba and C. FefFerman, On the collapse of tubes carried by 3D incompressible flows, Comm. Math. Phys., 222 (2001), pp. 293-298.

[20] D. Cordoba and C. Fefferman, Potato chip singularities of $3 D$ flows, SIAM J. Math. Anal., 33 (2001), pp. 293-298.

[21] Jian Deng, Thomas Y. Hou and Xinwei Yu, Geometric properties and non-blowup of 3-D incompressible Euler flow, 2004.

[22] P. G. Drazin and W. H. Reid, Hydrodynamic Stability, Cambridge U. Press, 1981.

[23] Weinan E and C. W. Shu, Numerical study of the small scale structures in Boussinesq convection, Phys. Fluids A, 6 (1994), pp. 49-58.

[24] Gregory Eyink, Energy dissipation without viscosity in ideal hydrodynamics. 1. Fourier analysis and local energy transfer, Physica D, 78 (1994), pp. 222-240.

[25] Gregory Eyink, Besov spaces and the multifractal hypothesis, J. Stat. Phys., 78 (1995), pp. 353-375.

[26] Akio Fukuyu and Tsutomu Arai, Singularity formation in three-dimensional inviscid flow, Fluid Dynamics Research, 7 (1991), pp. 229-240.

[27] J. D. GibBon, A quaternionic structure in the three-dimensional Euler and ideal magnetohydrodynamics equations, Physica D, 166 (2002), pp. 17-28.

[28] J.D. Gibbon, J. Gibbons And M. Heritage, A logarithmic 3D Euler inequality, Phys. Fluids, 9 (1997), pp. 471-472.

[29] R. Grauer, C. Marliani and K. Germaschewski, Adaptive mesh refinement for singular solutions of the incompressible Euler equations, Phys. Rev. Lett., 80 (1998), pp. 4177- 
4180.

[30] R. Grauer and T. Sideris, Numerical computation of $3 D$ incompressible ideal fluids with swirl, Phys. Rev. Lett., 67 (1991), pp. 3511-3514.

[31] X. Y. HE, Self-similar singularities of the 3D Euler equations, Appl. Math. Lett., 13 (2000), pp. $41-46$.

[32] Robert M. KERR, Evidence for a singularity of the three-dimensional, incompressible Euler equations, Phys. Fluids A, 5 (1993), pp. 1725-1746.

[33] T. Ogawa And Y. TANiuchi, On blow-up criteria of smooth solutions to the 3-D Euler equations in a bounded domain, J. Diff. Equations, 190 (2003), pp. 39-63.

[34] K. Ohkitani And J. D. GibBon, Numerical study of singularity formation in a class of Euler and Navier-Stokes flows, Phys. Fluids, 12 (2000), pp. 3181-3194.

[35] L. Onsager, Statistical Hydrodynamics, Nuovo Cimento (Supplemento), 6 (1949), pp. 279-287.

[36] R. B. PLEz, Extended series analysis of full octahedral flow: numerical evidence for hydrodynamic blowup, Fluid Dyn. Res., 33 (2003), pp. 207-221.

[37] Alain Pumir and Eric D. Siggia, Collapsing solutions to the 3-D Euler equations, Phys. Fluids A, 2 (1992), pp. 220-241.

[38] Alain Pumir and ERIC D. Siggia, Development of singular solutions to the axisymmetric Euler equations, Phys. Fluids A, 4 (1992), pp. 1472-1491.

[39] M. Siegel, Singulariy formation on vortex sheets, in P. Compte, H. Moffatt, G. Zaslavsky and M. Tabor, editors, Topological Aspects of the Dynamics of Fluids and Plasmas, pp. 337-349, Kluwer, 1992.

[40] M. Siegel, A study of singularity formation in the Kelvin-Helmholtz instability with surface tension, SIAM J. Appl. Math., 55 (1995), pp. 865-891.

[41] Michael Siegel, An analytical and numerical study of singularity formation in the RayleighTaylor problem, PhD thesis, NYU, 1989.

[42] Eric D. Siggia, Collapse and amplification of a vortex filament, Phys. Fluids, 28 (1985), pp. 794-805.

[43] C. Sulem, P. L. Sulem And H. Frisch, Tracing complex singularities with spectral methods, J. Comp. Phys., 50 (1983), pp. 138-161.

[44] V. A. Zheligovsky, E. A. Kuznetsov and O. M. Podvigina, Numerical modeling of collapse in ideal incompressible hydrodynamics, JETP Lett., 74 (2001), pp. 367-370. 
R. E. CAFLISCH AND M. SIEGEL 